\section{SP0128 THE VARIOUS ROLES OF PATIENT PARTICIPATION IN HTA BY THE EXAMPLE OF IQWIG}

Virginia Seiffart. Institute for Quality and Efficiency in Health Care (IQWiG), Drug Assessment, 50670 Köln, Germany

Background: The involvement of affected persons in preparing systematic reviews and HTA represents an established international standard of benefit assessment. The Institute of Quality and Efficiency in Health Care (IQWiG) is a professionally independent scientific Institute founded within the framework of the German Health Care Reform of 2004 as an establishment of the Foundation for Quality and Efficiency in Health Care. IQWiG provides benefit assessments addressing issues concerning the statutory health insurance services. Its responsibilities include among others the preparation of scientific reports, expert opinions and comments on quality and efficiency issues taking age, gender, and personal circumstances into account.

Objectives: The goal of this presentation is to provide an overview on different paths of involvement of patients and affected persons at IQWiG.

Methods: IQWiG's legal basis and responsibilities are anchored in the Social Code Book Fifth Book - Statutory Health Insurance (SGB V) and have been adapted and extended several times in the course of further health care reforms. The Institute's work is commissioned by the Federal Joint Committee or by the Federal Ministry of Health. The information for this presentation stems from IQWiG's General Methods (currently in version 5.0), a publicly available paper provided on the Institute's website, which explains the groundwork for its assessments.

Results: The involvement of affected persons at IQWiG primarily takes place during the initial work on a report within the framework of patient-relevant outcomes and relevant subgroups. Moreover, involvement can also include partaking in hearings. Affected persons include in particular patients (represented by parents or relatives, when appropriate) as well as potential participants in prevention measures. Affected persons are found via the patient representation of the Federal Joint Committee, as well as national or local self-help organizations or groups, hospitals or medical practices, external experts or other routes. The involvement can consist of a personal consultation or providing information in writing (through questionnaires or reports on personal experience), in both cases with documenting potential conflicts of interest.

Conclusion: As representatives of patients or self-help groups are sometimes not patients themselves and cannot directly account for symptoms or their impact on the quality of life, focus is placed on involving persons directly affected. Different assessments require distinct types of involvement depending on the available time and the confidentiality of the topic.

Disclosure of Interests: None declared

DOI: 10.1136/annrheumdis-2019-eular.8585

\section{SP0129 A PATIENT'S VIEW ON PATIENT INVOLVEMENT}

\section{Souzi Makri. CYPLAR, Limassol, Cyprus}

\section{Background: • HTA as defined by the EUPATI:}

Health technology assessment aims to inform decision making by health care policy makers. It is a systematic process that considers health technologies (such as medicines) and can involve a review of: Clinical evidence compared to existing models of care, Cost effectiveness, Social and ethical impacts on the health care system and the lives of patients EUPATI Overarching principle: "We recommend close cooperation and partnership between the various stakeholders including healthcare professionals' organisations, contract research organisations, patients' and consumers' organisations, academia, scientific and academic societies, regulatory authorities and HTA bodies and the pharmaceutical industry. Experience to date demonstrates that the involvement of patients has resulted in increased transparency, trust and mutual respect between them and other stakeholders. It is acknowledged that the patients' contribution to the discovery, development and evaluation of medicines enriches the quality of the evidence and opinion available."

Objectives: Inform decision making by health care policy makers. It is a systematic process that considers health technologies (such as medicines) and can involve a review of:

1. Clinical evidence compared to existing models of care,

2. Cost effectiveness,

3. Social and ethical impacts on the health care system and the lives of patients

\section{EUPATI Overarching principle:}

"We recommend close cooperation and partnership between the various stakeholders including healthcare professionals' organisations, contract research organisations, patients' and consumers' organisations, academia, scientific and academic societies, regulatory authorities and HTA bodies and the pharmaceutical industry. Experience to date demonstrates that the involvement of patients has resulted in increased transparency, trust and mutual respect between them and other stakeholders. It is acknowledged that the patients' contribution to the discovery, development and evaluation of medicines enriches the quality of the evidence and opinion available."

Objectives: Areas of patient involvement: Data, Decision problem (context), Scientific judgment and analysis,Value, Recommendation, Decision, Gover nance EUPATI guidance: (an exercise with 150 responders from 39 countries (Academia, HTA agencies, Patients industry : Knowledge and experience of patients are unique and contribute to essential knowledge - Fairness : Patients have the same rights as any other stakeholders to be part of the process -Equity: patient perspective helps to seek equity, by bringing into the process the true needs of patients, versus fairness of distribution of resources -Legitimacy : facilitates those affected by HTA decisions to participate contributing to the transparency and equability and credibility of the process - Capacity building : addresses barriers and helps building capacity for patients and HTA Organizations to work together

Methods: EPF Survey contacted 2010-2011 which produced a report among HTA agencies, decision makers and patient Organizations. The aim of the research was to assess the state of patient involvement in HTA. Responses collected from 40 HTA agencies, 18 decision makers and 23 patient organizations through on line questionnaires. The type of involvement varies. Few HTA agen cies currently involve patients perspectives in their reports and contact formal evaluation of the impact of patient involvement in HTA. Out of the 40 HTA agencies that responded, nearly half of them (18) involve patients in HTA. Out of the 23 patient organisations that completed the questionnaire, 10 indicated that their organisations have been involved in HTA. Out of 18 decision-makers, four stated that they are planning to involve patients in decision-making for health technologies and three said that they already involve patients. On the other hand, five respondents stated that they do not intend to involve patients in the future and the remaining six either stated that they did not know or did not respond to the question.

Results: There are four main challenges according to all three stakeholders to involve patients in HTA: (1) lack of an agreed and good method to obtain/provide patient evidence, (2) not knowing the stage at which patient engagement is needed and most useful, (3) patient involvement process being time intensive and (4) lack of capacity (HTA agencies, decision-making bodies, patient organisations). These themes represent all different forms of capacity that need to be in place for a meaningful patient involvement in HTA. According to the responses from the HTA agencies, high to moderate involvement is mainly seen in the phases of diffusion and dissemination, assessment, production of information, and external review. Low to no involvement is seen in the phases of identification, filtration, and prioritization.

Conclusions: The role of patient is very important in HTA in all aspects of the process. Before the medicine is developed, within HTA and when recommendations are made.

As patients' life and health state will be a affected by new technology, their input is vital in all stages of evaluating a new technology or medicinal product. Patients should be part of the process to explain WHY and HOW important is the proposed technology for the health. If there no involvement in all stages there is a risk of no deciding on the most appropriate technology and for crucial needs remain unmet. Recommendations for patient Organizations and HTA agencies exist in the EPF guide

\section{REFERENCES:}

[1] Results of the EPF Survey

[2] Patient involvement in the HTA decision-making process

Disclosure of Interests: None declared

DOI: 10.1136/annrheumdis-2019-eular.8532

FRIDAY, 14 JUNE 2019

13:30:00 - 15:00:00

\section{Capillaroscopy II}

\section{SP0130 STANDARDISATION OF NORMAL VERSUS ABNORMAL AND PATHOLOGICAL CAPILLAROSCOPIC IMAGES}

V. Smith ${ }^{1,2,3}$, on behalf of the EULAR Study Group on Microcirculation in Rheumatic Diseases. ${ }^{1}$ Department of Rheumatology, Ghent University Hospital; ${ }^{2}$ Department of Internal Medicine, Ghent University, ${ }^{3}$ Unit for Molecular Immunology and Inflammation, VIB Inflammation Research Center (IRC), Ghent, Belgium

Medical doctors frequently get patients with Raynaud's phenomenon (RP), a frequent symptom in the general population, referred. The importance of 
distinguishing normal capillaroscopic findings from (pathognomonic) abnormal (pathological) findings (scleroderma pattern), lies in the fact that this distinction allows the differentiation between a primary RP (not connected to any connective tissue disease [CTD]) from a secondary RP due to systemic sclerosis (SSc) and diseases of the scleroderma spectrum.

What is normal in primary RP?

A normal capillaroscopic pattern, by qualitative assessment, is characterized by a homogeneous distribution of hairpin shaped capillaries as a "comb-like structure", with a density of $>7$ capillaries per $\mathrm{mm}$, with a normal dimension and absence of large hemorrhages Yet, there exists a wide intra- and inter-individual variety in a normal population which will be discussed in this session.

What is pathognomonic abnormal in patients with RP due to SSc?

Patients with the RP who have an underlying clinically recognizable (= with skin involvement) SSc show a very characteristic combination of capillary abnormalities in the nailfold, which can easily be assessed through qualitative assessment (= pattern recognition). Maricq et al. described last century, with the widefield technique (magnification X12-14) the scleroderma pattern. This pathognomonic combination contains the following: a striking widening of all three segments of the capillary loop (arterial, venous and intermediate), loss of capillaries and disorganization of the nailfold capillary bed. Many branched "bushy" capillaries may also be observed.

In 2000, Cutolo et al. qualitatively assessed the nailfolds of an SSc cohort with patients fulfilling the American College of Rheumatology (ACR) criteria for SSc with the nailfold videocapillaroscopic (NVC) technique (magnification X200). According to the different proportions of the hallmark parameters of the scleroderma pattern (giants, capillary loss, hemorrhages and abnormal shapes: (neo) angiogenesis, Cutolo et al. defined three patterns "early", "active" and "late".

The central role of capillaroscopy in distinction between a primary and secondary RP due to SSc is reflected by the fact that capillaroscopy is one of the new ACR/ EULAR criteria for classifying a patient as having SSc.

In this lecture the standard "FAST TRACK" recognition system of the EULAR Study Group on Microcirculation in Rheumatic Diseases to discern scleroderma patterns from non-scleroderma patterns will be taught to the attendees.

Suggested further reading:

[1] Smith V, et al. Rheumatology (Oxford). 2016;55(5):883-90.

[2] Cutolo M, et al. Rheumatology (Oxford). 2018;57(4):757-759.

[3] Cutolo M, et al. Autoimmun Rev. 2018;17(4):344-352.

Disclosure of Interests: None declared

DOI: 10.1136/annrheumdis-2019-eular.8591

\section{SP0131 RELIABILITY IN THE LAST 10 YEARS: CAPILLAROSCOPIC CHARACTERISTICS VERSUS INSTRUMENTAL DETECTION}

Ariane Herrick. University of Manchester, Salford Royal Hospital NHS Foundation Trust, Division of Musculoskeletal and Dermatological Sciences, NIHR Manchester Musculoskeletal Biomedical Research Centre, Manchester, United Kingdom

Background: With nailfold capillaroscopy there are different aspects of reliability to consider, including reliability of qualitative image grading (e.g. 'early', 'active' and 'late') and also of semi-quantitative and quantitative measures including capillary density, apical width and presence of giant capillaries. The reliability of image acquisition (i.e. test-retest reliability) is especially important if nailfold capillaroscopic parameters are to be used in longitudinal studies (e.g. clinical trials) which involve acquiring repeat images over time. Lastly when assessing reliability, it is important to recognise that the nailfold capillaries cannot always be clearly seen, and cannot therefore be evaluated.

Objectives: To highlight the different aspects of reliability relating to nailfold capillaroscopy and how these have been addressed in studies over the last 10 years.

Methods: Review of recent studies.

Results:

1. Intra-observer reliability has been shown in several studies to be higher than inter-observer.

2. Assessment of 'evaluability' varies between observers and therefore also needs to be taken into account when assessing reliability.

3. Subject to evaluability, certain parameters demonstrate high intra- and inter-observer reliabilities. Reliability differs across different capillaroscopic parameters.

Conclusion: Recent studies examining reliability of capillaroscopy suggest that certain parameters, including image grade, capillary density and apex width have high intra-and inter-observer reliabilities (subject to nailfold image evaluability, which remains a major challenge). Standardised training is likely to improve reliability.

\section{REFERENCES}

[1] Murray AK, Vail A, Moore TL, et al. The influence of measurement location on reliability of quantitative videocapillaroscopy in patients with SSc. Rheumatol 2012; 51 : 1323-30.

[2] Overbury R, Murtaugh MA, Fisher A, et al. Primary care assessment of capillary abnormalities in patients with Raynaud's phenomenon. Clin Rheumatol 2015; 34: 2135-40.

[3] Smith V, Beeckman S, Herrick AL, et al. An EULAR study group pilot study on reliability of simple capillaroscopic definitions to describe capillary morphology in rheumatic diseases. Rheumatol 2016; 55: 883-90.

[4] Dinsdale G, Moore T, O'Leary N, et al. Intra-and inter-observer reliability of nailfold videocapillaroscopy - A possible outcome measure for systemic sclerosis-related microangiopathy. Microvascular Research 2017; 112: 1-6.

[5] Dinsdale G, Moore T, O'Leary N, et al. Quantitative outcome measures for systemic sclerosis-related microangiopathy - reliability of image acquisition in nailfold capillaroscopy. Microvascular Research 2017;113:56-9.

[6] Boulon C, Devos S, Mangin M, et al. Reproducibility of capillaroscopic classifications of systemic sclerosis: results from the SCLEROCAP study. Rheumatology 2017; 56: 1713-20.

[7] Cutolo M, Melsens K, Herrick AL, et al. Reliability of simple capillaroscopic definitions in describing capillary morphology in rheumatic diseases. Rheu matology (Letter) 2018; 57: 757-9.

Disclosure of Interests: None declared

DOI: 10.1136/annrheumdis-2019-eular.8499

\section{SP0132 METHODS AND TOOLS FOR QUANTIZATION OF CAPILLAROSCOPIC MORPHOLOGICAL CHANGES}

Francesca Ingegnoli. Academic Rheumatology Unit Universita degli Studi di

Torino, Academic Rheumatology Centre, Turin, Italy

Nailfold capillaroscopy is the one most used technique in both clinical and research settings by adult physicians and paediatric rheumatologists to assess patients with Raynaud's phenomenon as shown by an international survey on non-invasive techniques to assess the microcirculation performed under the aegis of members of the EULAR Study Group on Microcirculation in Rheumatic diseases [1].

Nailfold capillaroscopy is a simple non-invasive imaging technique mainly used to observe capillaries on the skin surface. After application of a drop of immersion oil, capillaries can be observed with a magnification lens because they run parallel to the epidermis at the nailbed area [2]

A number of different instruments can be used to perform the exam. They have different characteristics in terms of their cost, quality of images, magnifications, training period, portability, software for image analysis and storage.

Some of these instruments can be used both in clinical and research settings such as the stereomicroscope and the videocapillaroscope. The stereomicroscope allows the widefield visualization of the nailfold with low magnifications, the training is relatively short, but the examination is difficult to perform in patients with digital flexion contractures.

There appears to be consensus regarding the use of videocapillaroscopy that allows a detailed visualization of capillary morphology using higher magnifications (100-300x). Contact probe with polarized light microscopy permits easier observation of the skin surface, and the training period is briefer. Specific software are available for images analysis, storage, and complete medical reports (text + images) can be produced. By contrast, in a clinical setting, nailfold capillaries can generally be visualized using more simple, but also efficient tools such as a dermatoscope USB microscope, ophtamloscope or smartphone device. The quality of images can be quite good, although the lower magnification means that some details are unlikely to be seen, and they often lack the possibility of image storage and measurement. In particular, the dermatoscope with magnification of the order of $x 10$ is a small, inexpensive and easily portable piece of equipment that has been suggested to be comparable to videocapillaroscopy in routine clinical practice. As the study of capillary morphology provides clinically relevant information in the management of patients with scleroderma-spectrum diseases, the development of specific software to standardize and automatize the analysis is ongoing [3-4].

\section{REFERENCES:}

[1] Ingegnoli F, Smith V, Sulli A, Cutolo M. Capillaroscopy in Routine Diagnostics: Potentials and Limitations. Curr Rheumatol Rev. 2018 20;14(1):5-11

[2] Ingegnoli F, Ughi N, Dinsdale G, Orenti A, Boracchi $\mathrm{P}$, Allanore $\mathrm{Y}$, et al.; EULAR Study Group on Microcirculation in RheumaticDiseases. An international SUrvey on non-iNvaSive tecHniques to assess the mlcrocirculation in patients with RayNaud's phEnomenon (SUNSHINE survey). Rheumatol Int. 2017;37(11):1879-1890 